\title{
Severe acute malnutrition as a major predictor for poor nutritional outcome among adult HIV patients treated with FPB program: a case-control study
}

\author{
Nigest Abebaw*, Mulualem Endeshaw**, and Mamaru Ayenew***
}

ABSTRACT
INTRODUCTION
Different studies had explained the treatment outcomes of the food by
prescription (FBP) program among people living with HIV (PLHIV). This
study aimed to assess factors affecting nutritional treatment outcome
among PLHIV using FBP.

\section{METHODS}

An institution-based unmatched case control study was conducted from August 1 to September 30, 2017 in public health facilities. A total of 566 samples (283 cases and 283 controls) was drawn using random sampling technique. Data were collected from FBP, ART/PRE-ART registers and client's follow up charts. Bivariate and multivariate logistic regression analyses were conducted to estimate the relationship of the independent variables with the outcome variable, and a p-value $<0.05$ was considered as statistically significant at $95 \%$ confidence level. All statistical analysis was performed using SPSS version 23.

\section{RESULTS}

In this study severe acute malnutrition at admission ( $\mathrm{AOR}=4.45 .95 \% \mathrm{CI}$ : 2.63-7.55), moderate acute malnutrition at admission ( $\mathrm{AOR}=3.8,95 \% \mathrm{CI}$ : 2.18-6.67), lack of regular follow up ( $\mathrm{AOR}=3.81,95 \% \mathrm{CI}: 2.18-6.67)$, low $\mathrm{CD} 4$ count below $100(\mathrm{AOR}=2.93,95 \% \mathrm{CI}: 1.19-7.20)$, hemoglobin value below 10mg/dL (AOR=2.72, 95\% CI: 1.17, 6.35), and male sex $(\mathrm{AOR}=1.77$, $95 \% \mathrm{CI}: 1.19,2.63$ ) were found to be significant predictors for poor nutritional treatment outcome.

\section{CONCLUSIONS}

Severity of malnutrition was the major predictor for poor nutritional treatment outcome of patients who were on FBP program. The policy makers can use the information to improve HIV and nutrition interventions based on the result.

Keywords: Food by prescription, PLWHA, treatment outcome, Bahir Dar
*Monitoring and Evaluation coordinator at Plan International Ethiopia, Bahir Dar, Ethiopia

**Lecturer, Research and Publication Officer, Graduate Program Coordinator, Rift Valley University, Lancha Campus, Addis Ababa, Ethiopia

***Monitoring, Evaluation and Research, Amref Health Africa in Ethiopia, Addis Ababa, Ethiopia

\section{Correspondence:}

\section{Mulualem Endeshaw}

Lecturer, research and publication officer, Graduate Program Coordinator of Rift Valley University, Lancha Campus, Addis Ababa, Ethiopia Email:emulualem@gmail.com ORCID ID: orcid.org/0000-0001-

7773-9469

Date of first submission, November 8, 2018

Date of final revised submission, June 21, 2019

Date of acceptance, June 24, 2019

This open access article is distributed under a Creative Commons AttributionNon Commercial-Share Alike 4.0 International License

Cite this article as: Abebaw N, Endeshaw M, Ayenew M. Severe acute malnutrition as a major predictor for poor nutritional outcome among adult HIV patients treated with FPB program: a casecontrol study. Univ Med 2019;38:12130. doi: 10.18051/UnivMed.2019.v38. $121-130$ 


\section{INTRODUCTION}

The twin global epidemics of HIV infection and food scarcity disproportionately affect subSaharan Africa, and a significant proportion of patients who require antiretroviral therapy are malnourished because of a combination of HIVassociated wasting and inadequate nutrient intake. ${ }^{(1)}$ In resource-limited settings where poverty and food insecurity are common, provision of therapeutic and supplementary food products is a critical component of comprehensive care and support interventions. ${ }^{(2)}$ Nutritional interventions have been successful in the management of HIV and AIDS, and many patients enrolled into such programs have markedly improved both their body weight and general health status. ${ }^{(3)}$

Globally, an estimated 35.3 (32.2-38.8) million people were living with HIV at the end of 2015 and 70\% of people living with HIV (PLHIV) are located in sub-Saharan Africa. ${ }^{(4,5)}$ According to the national AIDS resource center 2014, adult HIV prevalence in Ethiopia is estimated at 1.2 percent. ${ }^{(6)}$ Currently in Ethiopia, there are about 789,960 adults living with HIV/AIDS. Of these, 204,000 (38.7\%) are found in Amhara National Regional State. ${ }^{(7)}$

Malnutrition among people living with HIV remains a major challenge in achieving the full impact of interventions aimed at improving the quality of life, productivity and survival. ${ }^{(2)}$ Over 850 million people worldwide and 200 million adults in Sub-Saharan Africa suffer from malnutrition. ${ }^{(8)}$ Malnutrition is also associated with faster disease progression, higher mortality rates, and suboptimal response to antiretroviral therapy (ART). ${ }^{(9)}$

Individuals at all stages of HIV disease are at risk of nutritional deficiency and nutritional status is a strong predictor of disease progression, survival and functional status during the course of the disease. ${ }^{(10)}$ Counseling and supporting people living with HIV is indicated as a solution to improve their nutritional status and their health. Attainment of good nutrition will contribute to the adoption of a positive attitude, which normally improves the quality of life for adults living with HIV. ${ }^{(11)}$

Different studies have reported that nutritional supplementation among PLHIV taken concurrently with ART have improved body mass index (BMI), ${ }^{(12-14)}$ survival, ${ }^{(15)} \mathrm{CD} 4$ count, ${ }^{(13)} \mathrm{HIV}$ viral load, ${ }^{(16)}$ immune response, ${ }^{(13,14)}$ quality of life, ${ }^{(14)}$ and adherence to antiretroviral therapy ${ }^{(17)}$

One way of addressing malnutrition among HIV/AIDS patients is through the Food by Prescription (FBP) program. ${ }^{(18)}$ The FBP program is one of the strategies that addresses under-nutrition among PLHIV through nutritional assessment, counseling, and support (NACS). ${ }^{(19)}$

The FBP program targets beneficiaries who are diagnosed to have either moderate acute malnutrition (MAM) or severe acute malnutrition (SAM) with two different products for a maximum of three or six months. Ready to use supplementary food (RUSF) is given for three months for MAM clients and ready to use therapeutic food (RUTF) is given for SAM cases. $^{(3)}$ In Amhara region, the FBP program has been implemented in 125 public health facilities since 2011 including four health facilities in Bahir Dar town.

Even though the FBP nutrition treatment program is designed with the objective to improve the nutritional and functional outcomes of PLHIV the intended outcome (recovery from malnutrition) is low. ${ }^{(3,20)}$ Studies conducted in Amhara region, ${ }^{(7)}$ Addis Ababa ${ }^{(18)}$ and Kenya (21) have reported low FBP recovery rates of $41 \%, 36.3 \%$ and $40.6 \%$ respectively. All these studies revealed that the recovery rate from malnutrition was below $50 \%$ and still remained to be a challenge. In relation to this there are previous studies conducted to assess the effectiveness of prescribed food on recovery from malnutrition, ${ }^{(3)}$ factors influencing adherence to the food by prescription program, ${ }^{(18)}$ nutrition outcomes of HIV-infected malnourished adults treated with ready-to-use therapeutic food ${ }^{(20)}$ and food insecurity and 
factors associated with malnutrition. ${ }^{(22)}$ The above studies focused on the outcome of patients treated with FBP and factors associated with malnutrition but not specifically on factors associated with treatment failure. Besides, studies that explore factors associated with nutritional treatment failure after FBP among adult PLHIV are limited in Ethiopia. Therefore this study tried to find out the factors contributing to treatment failure among adult PLHIV in Bahir Dar town health facilities with a different study design.

\section{METHODS}

\section{Study design and period}

This institution-based unmatched case control study was conducted from August 1, 2017 - September 30, 2017.

\section{Study area}

The study was conducted in four public health facilities of Bahir Dar town, that have been implementing FBP nutritional treatment program to PLHIV from March 2017 to December 2017. Bahir Dar is the capital of the Amhara Region, located $560 \mathrm{~km}$ from Addis Ababa. The FBP nutritional treatment program was started since 2011 in the Felege Hiwot Referral Hospital (FHRH) and Bahir Dar Health Center. The other two Han and Abay health centers have started FBP since 2012. The Felege Hiwot Referral Hospital serves about 15,961 PLHIV, of whom 3200 were enrolled into the FBP nutrition treatment program. There were 4, 524 PLHIV in Bahir Dar Health Center among whom 746 were enrolled into FBP. The numbers of PLHIV and FBP beneficiaries in Han HC and Abay HC were 3301,1514 and 254,321 respectively.

\section{Study population}

The study population were all adult nonpregnant, non-lactating PLHIV above the age of 18 years whose BMI was $<18.5 \mathrm{~kg} / \mathrm{m}^{2}$ and who were enrolled in to the FBP nutritional treatment program and had completed the treatment regime for three months (for MAM ) or six months (for SAM). Cases were PLHIV who did not recover from malnutrition (BMI $<18.5 \mathrm{~kg} / \mathrm{m} 2$ ) after treatment with RUTF/RUSF for a maximum of three months (MAM) or six months (SAM). Controls were PLHIV who recovered from malnutrition (BMI $\geq 18.5$ ) after treatment with RUTF/RUSF for a maximum of three (MAM) or six months (SAM).

\section{Sample size determination}

The sample size was determined using Epi Info Stat-Calc calculator for two populations by selecting power of $80 \%$, a significance level of $5 \%$, control to case ratio of $1: 1$ and taking poor ART adherence as a risk factor of recovery in the study conducted in Amhara region with AOR 2.14 for the proportion of exposure among recovered subjects of $9.7 \% .{ }^{(7)}$ Based on the above assumption, the total sample size required for cases and controls including 10\% anticipated dropouts was 566 or 283 pairs.

\section{Sampling technique}

After all cases and controls were sorted out from each health facility FBP register, simple random sampling technique was applied to select the required number of samples from each facility using a random number generator. The total sample size was proportionally allocated to the four health facilities based on their total number of recovered and non-recovered PLHIV.

\section{Study variables}

The outcome of the study was nutritional treatment outcome (recovered $=0$, unrecovered $=1$ ). Variables of interest collected were socioeconomic status, age, sex, marital status, religion, household size/number of people in the house, employment, educational status, residence, disclosure status about adherence to the FBP. The health status included CD4 count at entry, type of malnutrition, presence of opportunistic infections (OI's/TB, chronic diarrhea, candidiasis of the esophagus or lower respiratory tract, recurrent pneumonia), and other conditions such 
as Kaposi's sarcoma, lymphoma, and unexplained severe weight loss, WHO clinical stages I-IV, anti- retroviral therapy (ART) adherence, ART status, hemoglobin at admission, other FBP related factors, regular follow-ups, and type of prescribed commodity. In WHO clinical stage I the patient is asymptomatic and has persistent generalized lymphadenopathy, while in clinical stage II there is moderate unexplained weight loss of less than $10 \%$ of presumed or measured body weight. Clinical stage III is characterized by unexplained severe weight loss greater than $10 \%$ of presumed or measured body weight, unexplained chronic diarrhea for longer than one month, unexplained persistent fever above $37.6^{\circ} \mathrm{C}$ intermittent or constant, for longer than one month, while stage IV is characterized by HIV wasting syndrome or unexplained severe wasting, recurrent severe pneumonia, and extra pulmonary tuberculosis.

\section{Data collection method}

A standard checklist was used for extracting information from FBP registration book, pre- ART, ART, and patient follow up cards. The check list was checked for components that could capture the important information from the register and follow up cards. This form was developed using the standardized FBP follow up, pre ART, ART, and patient follow up cards employed by the ART clinic. The laboratory results of CD4 count recorded before enrolled in to FBP was used as a base line values. For those xith no pretreatment laboratory test, however, results obtained within one month of enrolment into FBP were considered as baseline values.

\section{Data analysis}

Descriptive analysis (frequency distribution, mean $( \pm \mathrm{SD})$ and percentages) were calculated to describe each socio-demographic variable. Based on this, univariate and multivariate logistic regressions were done to investigate associations of outcome of treatment (i.e. recovered and unrecovered) and different independent variables. P-value less than 0.05 was considered as statistically significant throughout the study.

\section{Ethical considerations}

Ethical clearance was gained from the Ethical review Committee of the Institute of Public Health, College of Medicine and Health Science, University of Gondar. Permission was also received from the regional health bureau under no. ARHB-307/17 and a copy of the letter given to the four health facilities, namely Felege Hiwot Referral Hospital (FHRH), Bahir Dar Health Center, Han Health Center, and Abay Health Center. Confidentiality was ensured from the investigator by using code numbers instead of names and the information collected was kept in a secured place.

\section{RESULTS}

\section{Socio-demographic characteristics of the study participants}

The study included a total of 566 (283 cases and 283 controls) eligible patients living with HIV/ AIDS recorded on food by prescription nutrition treatment in Bahir Dar town health facilities.

Among those 566 patients, three hundred twelve $(55.1 \%)$ were female. Of these, $49.5 \%$ were cases and $60.8 \%$ were controls. The majority of the study participants $(83.4 \%)$ were Christians and the rest $16.6 \%$ were Muslims. Regarding the marital status, 232 (41.0\%), of patients were married. The residence of patients in the group of cases were similar to that of patients in the group of controls, $89.8 \%$ of the cases and $90.8 \%$ of the controls were urban dwellers. As to educational status the majority of patients in both case $(72.8 \%)$ and controls $(73.8 \%)$ had received primary education. Concerning employment, $72.8 \%$ of cases and $73.9 \%$ of controls were formally employed in private and governmental institutions. Finally the majority of participants in both the cases and controls group were living with a family size of 1-4 people (Table 1 ). 
Table 1. Socio-demographic characteristics of cases versus controls

\begin{tabular}{|c|c|c|c|}
\hline Variable & $\begin{array}{c}\text { Cases n=283 } \\
(\%)\end{array}$ & $\begin{array}{c}\begin{array}{c}\text { Controls n }=283 \\
(\%)\end{array} \\
\end{array}$ & p value \\
\hline \multicolumn{4}{|l|}{ Sex } \\
\hline Male & $143(50.5)$ & $111(39.2)$ & \multirow[t]{2}{*}{0.007} \\
\hline Female & $140(49.5)$ & $172(60.8)$ & \\
\hline \multicolumn{4}{|l|}{ Age (years) } \\
\hline$\leq 34$ & $170(60.1)$ & $152(53.7)$ & \multirow[t]{4}{*}{0.111} \\
\hline $35-44$ & $71(25.1)$ & $91(32.2)$ & \\
\hline $45-54$ & $31(11.0)$ & 35 (12.4) & \\
\hline $55^{+}$ & $11(3.9)$ & $5(1.8)$ & \\
\hline \multicolumn{4}{|l|}{ Residence } \\
\hline Urban & $254(89.8)$ & $257(90.8)$ & \multirow[t]{2}{*}{0.672} \\
\hline Rural & $29(10.2)$ & $26(9.2)$ & \\
\hline \multicolumn{4}{|l|}{ Marital status } \\
\hline Not married & $68(24.0)$ & $66(23.3)$ & \multirow[t]{5}{*}{0.982} \\
\hline Married & $114(40.3)$ & $118(41.7)$ & \\
\hline Separated & $18(6.4)$ & $16(5.7)$ & \\
\hline Divorced & $59(20.8)$ & $61(21.6)$ & \\
\hline Widowed & $24(8.5)$ & $22(7.8)$ & \\
\hline \multicolumn{4}{|l|}{ Education } \\
\hline Primary & $206(72.8)$ & $209(73.8)$ & \multirow[t]{3}{*}{0.863} \\
\hline Secondary & $60(21.2)$ & $60(21.2)$ & \\
\hline Higher & $17(6.0)$ & $14(5.0)$ & \\
\hline \multicolumn{4}{|l|}{ Employment status } \\
\hline Employed & $206(72.8)$ & $209(73.9)$ & \multirow[t]{2}{*}{0.394} \\
\hline Un employed & $77(27.3)$ & $74(26.1)$ & \\
\hline \multicolumn{4}{|l|}{ Religion } \\
\hline Christian & $261(84.1)$ & $264(81.5)$ & \multirow[t]{2}{*}{0.822} \\
\hline Muslim & $22(15.9)$ & $19(18.5)$ & \\
\hline \multicolumn{4}{|l|}{ Household size } \\
\hline $1-4$ & $236(83.4)$ & $241(85.2)$ & \multirow[t]{3}{*}{0.641} \\
\hline $4-8$ & 45 (15.9) & $40(14.1)$ & \\
\hline$\geq 8$ & $2(0.7)$ & $2(0.7)$ & \\
\hline
\end{tabular}

* Christian (Orthodox, Protestant, Catholic)

\section{Factors associated with treatment outcome}

The bivariate analysis in this study revealed that there were several factors as predictors of food by prescription treatment outcome, i.e. sex, malnutrition status, failure of regular follow up, WHO clinical stage, CD4 count, and hemoglobin level, which were found to be predictors of food by prescription treatment success. When we look at the crude odds ratio (COR) of the covariates, males were 1.58 times more likely not to recover than females $(\mathrm{COR}=1.58,95 \%$ CI: 1.13-2.21). Malnutrition status is the other independent factor identified in this study, since patients who were diagnosed to have SAM at base line were 8.1 times more likely not to respond to therapeutic food than patients who had a diagnosis of mild malnutrition $(\mathrm{COR}=8.1$, 95\% CI: 3.84-17.01). Similarly, patients who were diagnosed to have moderate malnutrition were at higher risk of not recovering than did those with mild malnutrition $(\mathrm{COR}=6.25,95 \%$ CI: 4.25-9.18). Participants who had a CD4 count below 100 were 2.30 times more likely not to respond to therapeutic food than participants who had a CD4 count above 350 . Furthermore, patients who failed to have regular follow up, WHO clinical stage three, hemoglobin level below $10 \mathrm{mg} / \mathrm{dl}(\mathrm{COR}=3.63,95 \%$ CI: 2.24 5.87), (COR=1.68, 95\% CI: 1.09-2.60), $(\mathrm{COR}=2.77,95 \% \mathrm{CI}: 1.35-5.67)$ respectively, were also associated with increased risk of no recovery from malnutrition (Table 2 ). 
Table 2. Bivariate and multivariate logistic regression analysis of covariates with treatment outcome of FBP program participants

\begin{tabular}{|c|c|c|c|c|c|}
\hline Variable & $\begin{array}{c}\text { Cases } \\
(\%)\end{array}$ & $\begin{array}{c}\text { Controls } \\
(\%)\end{array}$ & COR & AOR & $\begin{array}{c}\text { p- } \\
\text { value }\end{array}$ \\
\hline \multicolumn{6}{|l|}{ Age (years) } \\
\hline$<34$ & $170(60.1)$ & $152(53.7)$ & 1 & & \\
\hline $35-44$ & $71(25.1)$ & $91(32.2)$ & $0.7(0.48,1.02)$ & & \\
\hline $45-54$ & $31(11.0)$ & $35(12.4)$ & $0.79(0.47,1.35)$ & & \\
\hline$>55$ & $11(3.9)$ & $5(1.8)$ & $1.97(0.67,5.79)$ & & \\
\hline \multicolumn{6}{|c|}{ 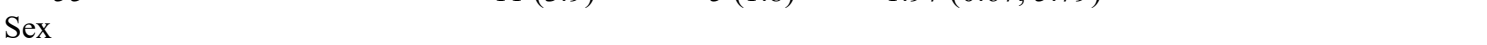 } \\
\hline Male & $143(50.5)$ & $111(39.2)$ & $1.58(1.13,2.21)$ & $1.77(1.19,2.63)$ & 0.005 \\
\hline Female & $140(49.5)$ & $172(60.8)$ & 1 & & \\
\hline \multicolumn{6}{|l|}{ Residence } \\
\hline Urban & $254(89.8)$ & $257(90.8)$ & $1.13(0.65,1.98)$ & & \\
\hline Rural & $29(10.2)$ & $26(9.2)$ & 1 & & \\
\hline \multicolumn{6}{|l|}{ Education } \\
\hline Primary & $206(72.8)$ & $209(73.9)$ & 1 & & \\
\hline Secondary & $60(21.2)$ & $60(21.2)$ & $1.02(0.68,1.52)$ & & \\
\hline Higher & $17(6.0)$ & $14(5.0)$ & $1.23(0.59,2.56)$ & & \\
\hline \multicolumn{6}{|l|}{ Disclosure } \\
\hline Yes & $224(79.2)$ & $225(79.5)$ & 1 & & \\
\hline No & $59(20.8)$ & $58(20.5)$ & $1.02(0.68,1.54)$ & & \\
\hline \multicolumn{6}{|l|}{ Type of malnutrition } \\
\hline Severe acute malnutrition & $34(12.0)$ & $10(3.5)$ & $8.10(3.84,17.01)$ & $4.45(2.63,7.55)$ & 0.0001 \\
\hline Moderate acute malnutrition & $160(56.5)$ & $61(21.6)$ & $6.25(4.25,9.18)$ & $3.81(2.18,6.67)$ & 0.0001 \\
\hline Mild malnutrition & $89(31.4)$ & $212(74.9)$ & 1 & & \\
\hline \multicolumn{6}{|l|}{ Type of commodity } \\
\hline RUTF & $159(56.2)$ & $141(49.8)$ & $1.29(0.93,1.79)$ & & \\
\hline RUSF & $124(43.8)$ & $142(50.2)$ & 1 & & \\
\hline \multicolumn{6}{|l|}{ Presence of OI } \\
\hline Yes & $22(7.8)$ & $22(7.8)$ & $1(0.54,1.85)$ & & \\
\hline No & $261(92.2)$ & $26(92.2)$ & 1 & & \\
\hline \multicolumn{6}{|l|}{ Regular follow up } \\
\hline Yes & $207(73.1)$ & $257(90.8)$ & 1 & & \\
\hline No & $76(26.9)$ & $26(9.2)$ & $3.63(2.24,5.87)$ & $3.81(2.18,6.67)$ & 0.0001 \\
\hline \multicolumn{6}{|l|}{ Employment } \\
\hline Yes & $206(72.8)$ & $209(73.9)$ & 1 & & \\
\hline No & $77(27.3)$ & $74(26.1)$ & $1.06(0.73,1.53)$ & & \\
\hline \multicolumn{6}{|l|}{ WHO stage } \\
\hline WHO stage 1 & $55(19.4)$ & $75(26.5)$ & 1 & & \\
\hline WHO stage 2 & $80(28.3)$ & $85(30.0)$ & $1.28(0.81,2.04)$ & & \\
\hline WHO stage 3 & $127(44.9)$ & $103(36.4)$ & $1.68(1.09,2.60)$ & $\dagger$ & \\
\hline WHO stage 4 & $21(7.4)$ & $20(7.0)$ & $1.43(0.71,2.89)$ & & \\
\hline \multicolumn{6}{|l|}{ ART Status } \\
\hline On ART & $221(78.1)$ & $219(77.4)$ & $1.04(0.70,1.55)$ & & \\
\hline Pre ART & $62(21.9)$ & $64(22.6)$ & 1 & & \\
\hline \multicolumn{6}{|l|}{ ART Adherence $(n=440)$} \\
\hline Yes & 202 & 204 & 1 & & \\
\hline No & 18 & 16 & $1.13(0.61,5.41)$ & $\dagger$ & \\
\hline \multicolumn{6}{|l|}{ CD4 (cells/mm3 ) } \\
\hline$<100$ & $12(4.24)$ & $26(9.20)$ & $2.30(1.05,5.07)$ & $2.93(1.19,7.20)$ & 0.019 \\
\hline $100-200$ & $51(18.02)$ & $48(16.96)$ & $2.35(1.11,4.96)$ & $3.79(1.61,8.92)$ & 0.002 \\
\hline $200-350$ & $89(31.45)$ & $82(28.98)$ & $2.24(1.08,4.62)$ & $4.20(1.82,9.71)$ & 0.001 \\
\hline$\geq 350$ & $131(45.33)$ & $127(44.88)$ & 1 & 1 & \\
\hline \multicolumn{6}{|l|}{$\mathrm{Hb}$ level } \\
\hline$<10 \mathrm{~g} / \mathrm{dL}$ & $29(10.25)$ & $11(3.88)$ & $2.77(1.35,5.67)$ & $2.72(1.17,6.35)$ & 0.021 \\
\hline $10-11.99 \mathrm{~g} / \mathrm{dL}$ & $30(10.60)$ & $37(13.07)$ & $0.85(0.51,1.42)$ & $0.88(0.46,1.61)$ & 0.65 \\
\hline$>12 \mathrm{~g} / \mathrm{dL}$ & $224(79.15)$ & $235(83.01)$ & 1 & 1 & \\
\hline
\end{tabular}

$\uparrow$ dropped in the final model, RUSF: ready to use supplementary food; RUTF: ready to use therapeutic food. COR=Crude odds ratio, $\mathrm{AOR}=$ adjusted odds ratio, $\mathrm{OI}=$ Opportunistic Infection, $\mathrm{ART}=$ anti- retroviral therapy 


\section{Results of multivariate analysis}

Variables that were significant at $p$-value 0.2 in the simple binary logistic regression analysis were further examined in the multiple binary logistic regressions to determine the relative effects of predictors of treatment outcome among attendants of FBP.

Factors such as male sex, malnutrition status, failure of regular follow up, lower CD4 count, and hemoglobin value below $10 \mathrm{mg} / \mathrm{dL}$ remained significant in the multivariate analysis in this study. Males were 1.77 times more at risk of non-recovery compared to females (AOR 1.77, 95\% CI: 1.18-2.6). Participants who were diagnosed as SAM during admission had an almost 4.5 times increased risk of not responding to therapeutic food compared to participants with mild malnutrition (AOR 4.45, 95\% CI: 2.63-7.55). In addition, lower CD4 count had a statistically significant association with non-recovery after treatment with FBP, CD4 count $<100,100-200,200-350$, $(\mathrm{AOR}=2.93,95 \% \mathrm{CI}: 1.19,7.20),(\mathrm{AOR}=3.79$, 95\% CI: $1.61-8.92),(\mathrm{AOR}=4.20-95 \%$ CI: 1.82 9.71 ) respectively. On top of this, patients with hemoglobin value below $10 \mathrm{mg} / \mathrm{dL}$ were at almost three times higher risk of no recovery after treatment with FBP than their counterparts with a normal hemoglobin value.

Unlike other studies, the present study found that factors such as age, residence, education level, disclosure status, type of commodity, presence of OI's, employment status, functional status and ART status did not show any significant association with treatment outcome (Table 2).

\section{DISCUSSION}

The main finding of this study was that severity of malnutrition and low CD4 count were the two major predictors for poor nutrition treatment response. The results of our study are in line with those of other similar studies conducted in Mekele, ${ }^{(23)}$ Amhara region (7) and Sub-Saharan countries. ${ }^{(20)}$ In our study, patients who were diagnosed as SAM during admission were at almost 4.5 times increased risk of not responding to therapeutic food compared to participants with mild malnutrition. This highlights the need to reach malnourished HIV patients early with nutritional support in order to maximize the recovery rate from malnutrition. ${ }^{(3)}$ In addition, lower CD4 counts of $<200$ cells $/ \mathrm{mm}^{3}$ and 200 350 cells $/ \mathrm{mm}^{3}$ were 3.79 and 4.2 times more at risk of non-response, respectively, compared to the higher CD4 count of $>350$ cells $/ \mathrm{mm}^{3}$. The result of this study was scientifically plausible, since our immunity is nutrition-dependent, PLHIV have a compromised immunity due to the effect of HIV, so that they are at risk for opportunistic infection which further exacerbates the malnutrition status and end up with poor response to nutritional supplement. In addition, the low CD4 count results in recurrence of opportunistic infection, with imbalance of nutritional requirement and uptake and thus ineffective nutritional supplementation response. ${ }^{(18)}$

In our study, males were 1.77 times more at risk of non-recovery from malnutrition compared to females. The result of this study is in agreement with that of previous studies done in other parts of Ethiopia, such as in Mekele. ${ }^{(23)}$ SubSaharan African counties also showed that males were at increased risk of non-recovery from malnutrition. ${ }^{(20)}$ This may be due to the fact that males tend to access HIV care at a more advanced stage of the disease than females. In addition, females may have good compliance to nutrition and HIV care that predisposes females to improve the uptake. ${ }^{(3)}$ In contrast to this, other studies done in Kenya ${ }^{(20)}$ and Amhara region ${ }^{(7)}$ reported that females are at increased risk of nonresponse to nutrition treatment. This difference may be attributed to the difference in study design, educational status, or temporal variation in patient characteristics.

Patients who failed to have regular followup during the FBP treatment were 3.8 times more at risk of nonrecovery from malnutrition than those having regular follow-up. This finding is consistent with that of other studies conducted in 
Amhara region, showing that patients with poor RUTF adherence were at 11 times lower risk of BMI improvement. ${ }^{(7)}$ Other studies also reported that missing follow-up appointments increased the risk of poor nutrition treatment outcome, ${ }^{(18,20,24)}$ since patients missing appointments also miss their monthly doses and the counseling that is provided by the health workers, leading to poor treatment adherence.

Patients with a hemoglobin value below $10 \mathrm{mg} / \mathrm{dL}$ were at almost 2.7 times higher risk of non-recovery after treatment with FBP than their counterparts with a normal hemoglobin value. The result of this study is in line with the established facts in many ways: Anemia is a condition characterized by inadequate red blood cells (erythrocytes) or hemoglobin. ${ }^{(26)}$ Anemia occurs when the body lacks sufficient amounts of iron, resulting in reduced production of the protein hemoglobin. PLHIV with poor diet are most susceptible to the disease since malnutrition is known to be a leading factor for the occurrence of anemia. An inadequate intake of macro- and micronutrients can play a well-documented role in chronic anemia pathophysiology due to the aforementioned facts together with the result of this study, in that there is a strong association between anemia and malnutrition. ${ }^{(25)}$

In this study, age was not found to be a predictor of FBP treatment outcome. However, other studies undertaken in different settings have documented that old age is associated with increased risk of non-response. ${ }^{(23,24)}$ ART status and number of months on ART also did not show any significant association with FBP treatment outcome in this study. But patients on Pre-ART were at risk of non-recovery compared to those on ART, and those patients who stayed on ART for more than 6 months were at increased risk of non-recovery compared to patients on PreART. ${ }^{(21,25)}$

Education level of patients and presence of opportunistic infections (OI's) did not show an association with treatment of FBP outcome in this study, however patients with low level of education were 1.82 times more at increased risk of non-recovery compared to educated ones, besides patients with presence of opportunistic infections were at higher risk of non-recovery from malnutrition compared to those without opportunistic infections. ${ }^{(25)}$ This may be due to the fact that the higher the level of education, the higher the awareness about management of HIV, opportunistic infections and malnutrition.

WHO clinical stage of disease did not show a significant association with FBP treatment outcome in this study, however a study done in Mekele Northern Ethiopia reported that WHO clinical stages I and II were four times more likely to respond to ready-to-use therapeutic food than patients with WHO stages III and IV. ${ }^{(25)}$ The rest of the factors such as type of commodity, employment status, disclosure status, and residence were not associated with nutritional treatment outcome in this study and other studies conducted so far.

The main limitation of this study was its retrospective nature that limits its ability to gather data about factors that may influence failure to respond to ready-to-use food therapy. It is recommended that clinicians ought to encourage regular screening of nutritional status among PLHIV in order to identify malnourished individuals early to improve the outcome of FBP. Further longitudinal research is needed to understand the effect of dosage and compliance of individuals on FBP.

\section{CONCLUSION}

Severity of malnutrition and lower CD4 count were the most influencing factors of the outcome of ready-to-use therapeutic food by prescription. Hence encouraging regular and comprehensive nutritional screening on PLHIV and maximizing efforts to reach malnourished HIV patients early with nutritional support for recovery, besides strengthening the nutritional adherence counseling for patients on ready-touse therapeutic food, are crucial elements to improve the outcome of FBP. 


\section{ACKNOWLEDGMENT}

The authors would like to thank the Rift Valley University for sponsoring this study. The authors are also grateful to the Amhara regional health bureau, Felege Hiwot Referral Hospital, Bahir Dar Health Center, Han Health Center and Abay Health Center for facilitating the data collection process. We also extend our appreciation to our data collectors for their contribution in the data collection process.

\section{AUTHORS’ CONTRIBUTIONS}

NA has contributed to the design, data collection and write up of the study protocol, ME has contributed to the design, data analysis, and manuscript development and revisions, and MA has contributed to the revision of the manuscript. All authors have read and approved the final draft.

\section{COMPETING INTERESTS}

The authors declare that they have no competing interests.

\section{REFERENCES}

1. Koethe JR, Heimburger DC. Nutritional aspects of HIV-associated wasting in sub-Saharan Africa. Am J Clin Nutr 2010;91:1138S-42S. doi: 10.3945/ajen.2010.28608D.

2. Food and Nutrition Technical Assistance II Project (FANTA-2). Review of Kenya's Food by Prescription Program. Connecticut Ave., NW Washington, DC: Food and Nutrition Technical Assistance II Project (FANTA-2);2009.

3. Sadler K, Bontrager E, Rogers B, et al. Food by Prescription: measuring the impact and costeffectiveness of prescribed food on recovery from malnutrition and HIV disease progression among $\mathrm{HIV}+$ adult clients in Ethiopia. Boston: Feinstein International Center;2012.

4. UNAIDS. Global report: UNAIDS report on the global AIDS epidemic. Geneva: UNAIDS; 2013.

5. UNAIDS. People living with HIV: the gap report. Geneva: UNAIDS; 2014.

6. Ethiopian Health and Nutrition Research Institute Federal Ministry of Health. HIV related estimates and projections for Ethiopia. Addis Ababa,
Ethiopia: Ethiopian Health and Nutrition Research Institute;2012.

7. Gedefaw M, Tariku M. Determinates of regain in body mass index among malnourished Aids patients on therapeutic food in Amhara national regional state, Northwest Ethiopia: a retrospective cohort study. Open J Epidemiol 2015;5:122-8. doi: 10.4236/ojepi.2015.52016.

8. Sicotte M, Langlois ÉV, Aho J, et al. Association between nutritional status and the immune response in HIV + patients under HAART: protocol for a systematic review. Syst Rev 2014;3: 9. doi: 10.1186/2046-4053-3-9.

9. Swaminathan S, Padmapriyadarsini C, Yoojin L, et al. Nutritional supplementation in HIV-infected individuals in South India: a prospective interventional study. Clin Infect Dis 2010;51:517. doi: $10.1086 / 653111$.

10. Ivers LC, Cullen KA, Freedberg KA, et al. HIV/ AIDS, under- nutrition, and food insecurity. Clin Infect Dis 2009;49:1096-102. doi: 10.1086/605573.

11. Bontrager E, Sadler K. Early stages of a 'Food by Prescription' programme for HIV infected adults. ENN Strategy; 2011.

12. Ndekha MJ1, van Oosterhout JJ, Zijlstra EE, et al. Supplementary feeding with either ready-to-use fortified spread or corn-soy blend in wasted adults starting antiretroviral therapy in Malawi: randomized, investigator blinded, controlled trial. BMJ 2009; 338:b1867. doi: 10.1136/bmj.b1867.

13. Olsen MF, Abdissa A, Kæstel P, et al. Effects of nutritional supplementation for HIV patients starting antiretroviral treatment: randomised controlled trial in Ethiopia. BMJ 2014;348:g3187. doi: 10.1136/bmj.g3187.

14. Evans D, McNamara L, Maskew M, et al. Impact of nutritional supplementation on immune response, body mass index and bioelectrical impedance in HIV-positive patients starting antiretroviral therapy. Nutr J 2013;12:111. doi: 10.1186/1475-2891-12-111.

15. Lategan R, Steenkamp L, Joubert G, et al. Nutritional status of HIV-infected adults on antiretroviral therapy and the impact of nutritional supplementation in the Northern Cape Province, South Africa. S Afr J Clin Nutr 2010;23:197-201.

16. Rawat R, Kadiyala S, McNamara PE. The impact of food assistance on weight gain and disease progression among HIV-infected individuals accessing AIDS care and treatment services in Uganda. BMC Public Health 2010;10:316. DOI: https://doi.org/10.1186/1471-2458-10-316.

17. Tafese Z, Birhan Y, Abebe H. Nutritional care and support among adults living with HIV at Hawassa referral hospital, southern Ethiopia: a qualitative 
study. Afr JAIDS Res 2013;12:105-11. doi: 10.2989/ 16085906.2013.825301.

18. Kebede MA, Haidar J. Factors influencing adherence to the food by prescription program among adult HIV positive patients in Addis Ababa, Ethiopia: a facility-based, cross-sectional study. Infect Dis Poverty 2014;3:20. doi: 10.1186/ 2049-9957-3-20.

19. Moench-Pfanner R, Van Ameringen M. The Global Alliance for Improved Nutrition (GAIN): a decade of partnerships to increase access to and affordability of nutritious foods for the poor. Food Nutr Bull 2012;33(4Suppl):S373-80. doi: 10.1177/ $15648265120334 \mathrm{~S} 313$.

20. Ahoua L, Umutoni C, Huerga H, et al. Nutrition outcomes of HIV-infected malnourished adults treated with ready-to-use therapeutic food in subSaharan Africa: a longitudinal study. J Int AIDS Soc 2011;14:2. doi: 10.1186/1758-2652-14-2.

21. Nagata JM, Cohen CR, Young SL, et al. Descriptive characteristics and health outcomes of the food by prescription nutrition supplementation program for adults living with HIV in Nyanza Province, Kenya. PLoS One 2014;9:e91403. doi: 10.1371/journal.pone.0091403.
22. Gebremichael D, Hadush K, Kebede E, et al. Food insecurity, nutritional status, and factors associated with malnutrition among people living with HIV/AIDS attending antiretroviral therapy at public health facilities in West Shewa Zone, Central Ethiopia. Bio Med Res Intl 2018, Article ID 1913534. doi: 10.1155/2018/1913534.

23. Maldey B, Haile F, Shumye A. Outcome of ready to use food therapy among patients on HIV/ AIDS care in Mekelle hospital, Northern Ethiopia: retrospective cohort study. J AIDS Clin Res 2014;5:1-6. doi: 10.4172/2155-6113.1000268.

24. Bahwere P, Deconinck H, Banda T, et al. Effective therapeutic feeding with chickpea sesame based ready-to-use therapeutic food (CS-RUTF) in wasted adults with confirmed or suspected AIDS. World JAIDS 2011;1:169-81. doi: 10.4236/wja.2011. 14025.

25. Janz TG, Johnson RL, Rubenstein SD. Anemia in the emergency department: evaluation and treatment. Emerg Med Pract 2013;15:1-15. 